\title{
Two-hybrid reporter vectors for gap repair cloning
}

\author{
Jennifer I. Semple, Graham Prime², Lise J. Wallis², Christopher M. Sanderson ${ }^{1}$, and David Markie ${ }^{2}$
}

BioTechniques 38:927-934 (June 2005)

\begin{abstract}
Yeast two-hybrid analysis is a valuable approach to the discovery and characterization of protein interactions. We have developed vectors that can indicate the presence of an insert when used in two-hybrid bait and prey construction by gap repair cloning. The strategy uses a recombination cloning site flanked by sequences encoding the GAL4 activation and binding domains. After gap repair cloning in standard hosts carrying an ADE2 reporter gene, disruption of GAL4 by an insert can be identified by the development of red colony color, while empty vector plasmids produce white colonies. Function in yeast two-hybrid applications was initially validated using known interacting proteins in pair-wise analyses, and subsequently, the bait vectors were used in library screens with the mouse Mad2l2 and human Mccdl proteins, identifying a number of putative new interactions for these proteins. These vectors should facilitate high-throughput yeast two-hybrid screens in which large numbers of bait and prey constructs may be required.
\end{abstract}

\section{INTRODUCTION}

Since its description in 1989 (1), yeast two-hybrid analysis has become a widely used and invaluable tool for the identification of new protein interactions. Increasingly, two-hybrid analysis is being used in high-throughput approaches to address protein interactions on a global scale, and attempts at interaction analysis of entire viral (2), bacterial (3), yeast (4-6), and fruitfly proteomes (7) have been undertaken, with similar approaches in progress in the nematode Caenorhabditis elegans (8). To accommodate the scale of these analyses, significant methodological improvements have been required, including the development of hosts with multiple reporter genes to decrease false positives (9), mating-based approaches for screening libraries $(4,6,10-12)$, and the use of gap repair approaches to directly clone DNA fragments in yeast. This last approach, based on the ability of yeast to recombine homologous sequences in vivo (13), can be used to construct plasmids from DNA fragments with overlapping terminal sequences (14).
Sequences as short as $25-30$ bp can direct homologous recombination in yeast, enabling the addition of tags by PCR for cloning DNA fragments into linearized vectors (15). Gap repair cloning directly in a yeast host has clear advantages for large-scale yeast two-hybrid screens, where many bait and prey constructions may be required. However, an issue with using this methodology is the background of clones without inserts resulting from residual nonlinearized vector molecules. To expedite bait and prey cloning, we have developed vectors that can indicate the presence of an insert in standard yeast two-hybrid host strains. The strategy for insert indication is based on a disruption of function approach. Bait vectors were constructed by placing the GALA activation domain sequence downstream of a binding domain sequence, and prey vectors were constructed by placing the sequences in the opposite orientation. Both of these arrangements reconstitute GAL4 function from the empty vector that is capable of activating standard two-hybrid reporter genes. Insertion of appropriate DNA fragments at the cloning site disrupts GAL4 function, but maintains expression of fusion proteins similar to conventional yeast two-hybrid bait and prey vectors, respectively. The $A D E 2$ reporter gene, present in commonly used PJ69-4A and AH109 host strains, is most convenient to detect loss of GAL4 function as it can be scored directly by colony color.

\section{MATERIALS AND METHODS}

\section{Yeast Strains, Media, and Manipulation}

The two-hybrid yeast strain PJ694A (9) was used as a host, and strain PJ69-4 $\alpha$ was developed to provide a suitable mating partner with identical genotype. To achieve this, the mating type of PJ69-4A was switched by transient expression of the $\mathrm{HO}$-endonuclease from plasmid pGAL-HO (16). As expression of the $H O$-endonuclease from this plasmid is GAL4-dependent and the PJ69-4A host is null for this gene, the entire GAL4 gene was first cloned at the SalI site of pGAL-HO. Mating type switching was then carried

\footnotetext{
${ }^{1}$ MRC Rosalind Franklin Centre for Genomics Research, Cambridge, UK and ${ }^{2}$ University of Otago, Dunedin, New Zealand
} 
out as described previously (16), and the resulting strain was subsequently cured of the plasmid by growth in nonselective conditions to produce PJ69-4 $\alpha$.

Yeast were routinely grown in YPAD medium $(10 \mathrm{~g} / \mathrm{L}$ yeast extract, $20 \mathrm{~g} / \mathrm{L}$ peptone, $20 \mathrm{~g} / \mathrm{L}$ D-glucose, $50 \mathrm{mg} / \mathrm{L}$ adenine hemisulfate, $15 \mathrm{~g} / \mathrm{L}$ agar when required). For selection, $\mathrm{SD}$ medium was used $(6.7 \mathrm{~g} / \mathrm{L}$ yeast nitrogen base without amino acids, $20 \mathrm{~g} / \mathrm{L} \mathrm{D}$-glucose) with appropriate supplements for the PJ69-4A host to produce selective dropout media (20 $\mu \mathrm{g} / \mathrm{mL}$ adenine hemisulfate, $20 \mu \mathrm{g} / \mathrm{mL}$ uracil, $20 \mu \mathrm{g} / \mathrm{mL}$ L-histidine, $20 \mu \mathrm{g} / \mathrm{mL}$ L-tryptophan, $20 \mu \mathrm{g} / \mathrm{mL}$ L-methionine, $100 \mu \mathrm{g} / \mathrm{mL}$ L-leucine). For optimal growth and the development of red color, bait strains were grown on SD medium supplemented with $14 \mathrm{~g} / \mathrm{L}$ acid hydrolyzed casein (SD+C medium) and $20 \mu \mathrm{g} / \mathrm{mL}$ each of adenine hemisulfate and uracil. The lithium acetate method (17) was used, with minor modifications, for all yeast transformations.

\section{Construction of Vectors}

To produce the bait vector $\mathrm{pGBAD}$ $\mathrm{B}$, a DNA fragment that contained the GAL4 activation domain sequence was placed in-frame with the GAL4 DNA binding domain sequence in plasmid pGBD-C1 (9), and the multiple cloning site was replaced with a synthetic DNA fragment containing the $a t t B 1$ and $a t t B 2$ sequences flanking a $B a m \mathrm{HI}$ restriction site (Figure 1). This vector was designed for cloning DNA fragments that incorporate a termination codon at the end of an open reading frame. A matched non-indicator bait plasmid (pGBD-B) was constructed by directly replacing the cloning site of pGBD-C1 with the same synthetic DNA fragment.

A second indicator bait plasmid was constructed that relies on frameshifting rather than a stop codon to report the presence of an insert. This was achieved by replacing the cloning site of pGBAD-B with an alternative sequence that adds a single nucleotide (cytosine) between the recombination sites and a TA dinucleotide downstream that creates a stop codon in the appropriate reading frame (Figure 1). This plasmid was designated pGBAE-B.
In a similar approach for constructing insertional indicator vectors for prey cloning, the GAL4binding domain sequence was placed downstream of the activation domain sequence in pACT2 (BD Biosciences Clontech, Palo Alto, CA, USA), and the original cloning site was replaced as before to produce pACTBD-B. This vector relies on a termination codon at the end of the incorporated open reading frame to indicate insertion. To provide a matched non-indicator prey vector, the cloning site of $\mathrm{pACT} 2$ was also directly replaced with the new cloning site, producing pACT2-B.

A second indicator prey plasmid, pACTBE-B, which relies on a frameshift rather than a stop codon, was constructed in the same way as pGBAE-B, except that a TG rather than a TA dinucleotide was inserted to create the required stop codon (Figure 1).

To provide a plasmid capable of expressing an ochre termination suppressor in yeast, a 914-bp SalIHindIII fragment, containing the SUP4$o$ allele from pYAC4, was ligated into

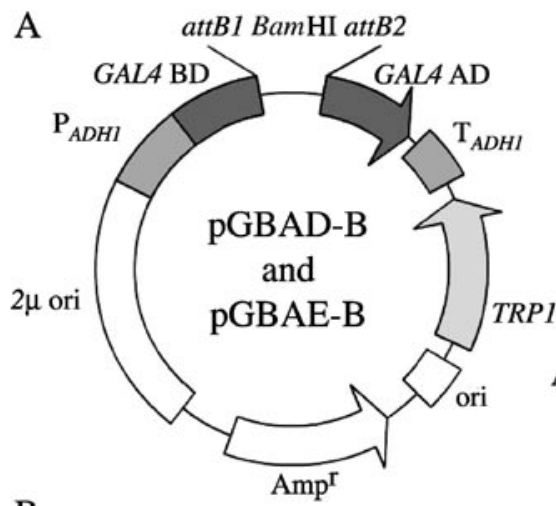

B

pGBD-B, pGBAD-B, pACT2-B, and pACTBD-B

GAA TTC ACA AGT TTG TAC AAA AAA GCA GGC TGG ATC CAC CCA GCT TTC TTG TAC AAA GTG GTC GAC
EcoRI
attBI

pGBAE-B

GAA TTC ACA AGT TTG TAC AAA AAA GCA GGC TGG AIC CCA CCC AGC TTT CTT GTA CAA AGT GGT CGA OTI
$\begin{gathered}\text { EcoRI } \\ \text { attBl }\end{gathered}$
After cloning
GAA TTC ACA AGT TTG TAC AAA AAA GCA GGC TGG NNN CAC CCA GCT TTC TTG TAC AAA GTG GTC GAC TAG

pACTBE-B

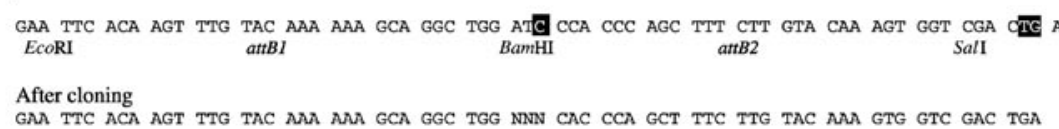

Figure 1. Two-hybrid vectors. (A) Vector structure. The two indicator bait vectors pGBAD-B and pGBAE-B are based on the pGBD-C1 vector and are identical in overall structure to one another (left). The non-indicator bait vector pGBD-B (not shown) is similar in structure but lacks the GAL4 activation domain. The two indicator prey vectors pACTBD-B and pACTBE-B (right) are based on pACT2. The non-indicator prey vector pACT2-B is similar but lacks the GAL4-binding domain sequence. (B) Vector cloning sites. The $a t t B 1$ and $a t t B 2$ sequences are incorporated in the cloning sites of all vectors and consequently are included as part of the A1 and A2 tags added to PCR fragments for cloning. In the indicator vectors, the cloning site maintains an open reading frame between the two GAL4 domain sequences, as indicated by the triplet spacing. For indicator function in the pGBAD-B and pACTBD-B vectors, an inframe stop codon (TAA) is incorporated at the end of the insert fragment to terminate translation of the fusion protein, thereby extinguishing GAL4 function. Indicator function for pGBAE-B and pACTBE-B is based on frameshifting. In these vectors, the addition of one nucleotide between the recombination sequences and two nucleotides downstream (shown in boxes) maintains the reading frame prior to cloning, but incorporation of an open reading frame by gap repair cloning (shown as NNN) shifts the reading frame with subsequent translation termination directly after the A2 recombination site. 
SalI-HindIII-digested YCplac111, a yeast-Escherichia coli shuttle vector that carries the yeast $L E U 2$ gene (18). After construction in E. coli, this plasmid (pSUP4) was transferred to the two-hybrid host strains for use as a mating partner to test for intact open reading frames from inserts cloned in pGBAD-B.

\section{Cloning of Open Reading Frames as Baits and Preys}

Gene fragments were obtained by PCR using Expand ${ }^{\mathrm{TM}} \mathrm{High}$ Fidelity DNA Polymerase (Roche Diagnostics, Indianapolis, IN, USA) from mouse or human first-strand cDNA with gene-specific primers that add short sequence tags. The A1 tag (5'-GAATTCACAAGTTTGTACAAAAAAGCAGGCTGG-3') was added in frame to sense primers, and the A2 tag (5'-GTCGACCACTTTGTACAAGAAAGCTGGGTG-3') was added in frame to antisense primers. An in-frame ochre stop codon was added to the antisense tag (5'-GTCGACCACTTTGTACAAGA A AGCTGGGTGTTA-3') when required for cloning in the pGBAD-B and pACTBD-B vectors. The resulting DNA fragments were cotransformed into yeast hosts with BamHI-linearized vector DNA and plated with selection to enrich for functional plasmid reconstruction by gap repair cloning. For bait cloning, the selective medium was $\mathrm{SD}+\mathrm{C}$ supplemented with uracil $(20 \mu \mathrm{g} /$ $\mathrm{mL})$ and limiting adenine $(20 \mu \mathrm{g} / \mathrm{mL})$, which allows selection for the TRPI gene on bait vectors but provides better color development in these host strains than standard -trp dropout medium. Standard -leu dropout medium with limiting adenine was used for prey cloning. Individual colonies were tested by PCR for the presence of an insert of the correct size, but were not further characterized by DNA sequencing.

\section{Pair-Wise Mating for Interaction Testing}

For interaction testing, single colonies from bait and prey constructions were suspended in sterile water, mixed in appropriate combinations, and $5-\mu \mathrm{L}$ aliquots were arrayed onto the surface of a YPAD plate for overnight growth at $30^{\circ} \mathrm{C}$. Following replica plating to -trp-leu dropout medium and growth to establish diploids, testing for reporter gene activation was achieved by further replica plating to -trp-leu-his and to -trp-leu-ade dropout medium.

\section{Two-Hybrid Library Screening}

Plasmid cDNA libraries in the vector $\mathrm{pACT} 2$ were obtained from $\mathrm{BD}$ Biosciences Clontech and transformed into yeast host strains. The resulting colonies were harvested, pooled, made to $15 \%$ glycerol, and frozen at $-70^{\circ} \mathrm{C}$ in aliquots of at least $10^{8}$ viable cells. For Mad212 screens, a single aliquot of frozen library was thawed, added to 100-200 mL YPAD broth, and grown overnight at $30^{\circ} \mathrm{C}$ with shaking. For Mccd1 screens, frozen aliquots $(5 \times$ $10^{8}$ viable cells) were used directly for mating without prior overnight growth in YPAD.

A pool of 6-12 individual colonies from bait constructions was made for library screens and grown overnight in $\mathrm{SD}+\mathrm{C}$ broth supplemented with 100 $\mu \mathrm{g} / \mathrm{mL}$ adenine hemisulfate and 20 $\mu \mathrm{g} / \mathrm{mL}$ uracil. For each screen, $5 \times 10^{8}$ freshly grown bait cells were mixed with a similar number of cells from the library culture and spread over a 140-mm diameter plate of YPAD agar. After $4-6 \mathrm{~h}$ incubation at $30^{\circ} \mathrm{C}$, the cells were harvested and spread over selective plates of -leu-trp-his dropout medium with a total surface area of around $2000 \mathrm{~cm}^{2}$. Colonies were picked after 3 days growth and checked for activation of second and third reporter genes (9). Confirmation of interactions was undertaken in a similar manner to that described by Petermann et al. (19). In brief, prey inserts were amplified using vector-specific primers with attached A1 and A2 tags, and "rescued" by gap repair cloning with BamHI-digested pACT2-B (a nonindicator vector) into a "naïve" host of appropriate mating type. No attempt was made to differentiate clones with inserts, and all colonies were mated to bait strains using a replica plating approach. Confirmation of a specific interaction in the yeast two-hybrid system was assumed if multiple rescued prey colonies from a primary clone demonstrated growth on -ade and -his dropout medium after mating with the original bait strain, but not with an unrelated bait strain. For clones that demonstrated a reproducible and specific yeast two-hybrid interaction, interaction sequence tags (ISTs) were then obtained by sequencing the PCR fragments from prey inserts, and the corresponding genes were identified by searching nucleic acid databases.

\section{RESULTS}

\section{Strategy for Vector Design}

In addition to the reconstruction of GAL4 function to allow a simple assay for disruption by insertion, some consideration was given to the sequences used for recombination cloning. A cloning site common to all vectors, which included the $a t t B 1$ and $a t t B 2$ sequences (Figure 1B), was used so that appropriately tagged DNA fragments could be readily cloned as both baits and preys in yeast, as well as providing compatibility with the Gateway ${ }^{\mathrm{TM}}$ system (Invitrogen, Carlsbad, CA, USA) for cloning the same fragments into E. coli.

Two alternative approaches were used to produce termination after translation of the insert fragment and prior to the downstream GAL4 domain in the vectors. In the first, a stop codon was incorporated at the end of open reading frames during $\mathrm{PCR}$, and the resulting DNA fragments were cloned into the pGBAD-B and pACTBD-B indicator vectors or into the $\mathrm{pGBD}-\mathrm{B}$ and pACT2-B non-indicator vectors. This approach has the advantage that the $\mathrm{C}$ terminus of the fusion protein is completely defined and contains no extra peptide tag that may interfere with interactions. In addition, subsequent mating to a yeast strain expressing an ochre termination suppressor allele from the pSUP4 plasmid (see the Materials and Methods section) can be used to confirm that the cloned insert encodes an open reading frame through restoration of GAL4 function, although this was not utilized for the routine cloning of baits and preys described in this study. However, one disadvantage of incorporating a stop codon 
in the insert is that it limits utility when cloning the same fragment in Gateway destination vectors designed to express $\mathrm{C}$-terminal fusion proteins. For this reason, a second approach was developed that relies on insertdependent frameshifting in the vectors for reporting insertion, but maintains the reading frame between the insert and the downstream attB2 sequence (Figure 1B) for Gateway cloning.

\section{Performance in Gap Repair Cloning}

As expected, the use of indicator vectors for gap repair cloning of PCR fragments as baits and preys produced a mixture of red and white colonies on appropriate medium containing limiting concentrations of adenine (Figure 2). The development of red color was improved by including acid-hydrolyzed casein in the medium $(\mathrm{SD}+\mathrm{C})$ when cloning baits, presumably because this supplement provides amino acids that are limiting, but not essential, for growth of the host strains. Similar supplementation was precluded for prey cloning, as selection for the LEU2 gene can not be applied, but color development was adequate on standard dropout medium to allow differentiation of colonies.

To determine the efficacy of red colony color as an indication of insertion, individual clones were amplified using the A1 and

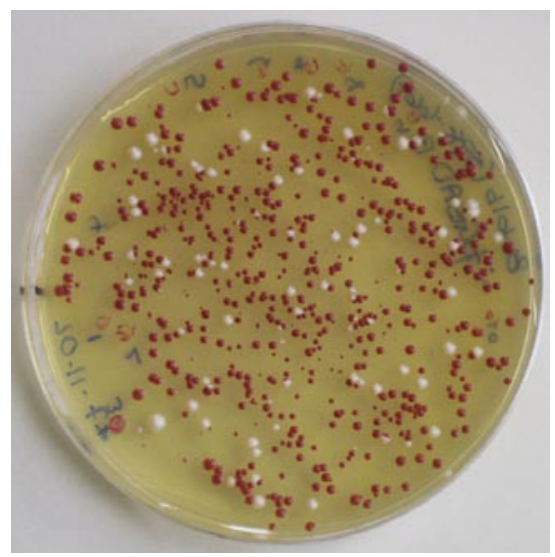

Figure 2. Colony color as an indicator of insert status in the bait vector pGBAD-B. Disrupted $G A L 4$ function fails to activate the $A D E 2$ reporter gene resulting in the production of red colony pigmentation in clones that contain inserts. White colonies represent clones with intact GAL4 function owing to the absence of inserts.
A2 sequences as PCR primers and analyzed on agarose gels. In one such analysis, a total of 96 colonies from six different open reading frames cloned in the pGBAD-B plasmid were examined (see Figure S1 in the Supplementary material that can be found online atwww.BioTechniques.com). Ninetyfour colonies produced a fragment of the expected size, one colony produced no fragment from PCR, and one colony produced two fragments, suggesting a greater than $95 \%$ cloning efficiency when red colonies were selected. This result was representative of most gap repair cloning experiments, although

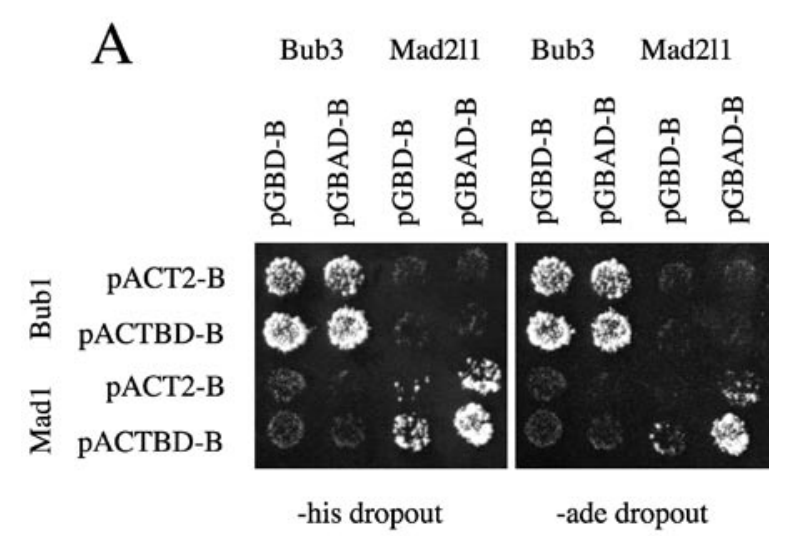

B

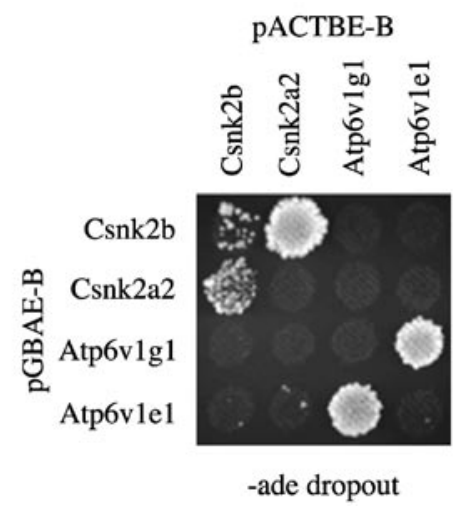

Figure 3. Replication of known protein interactions. (A) Mouse Bub3 and Mad2ll genes were cloned as baits into pGBD-B and pGBAD-B. Fragments of mouse Bubl and Madl genes were cloned as preys in $\mathrm{pACT} 2-\mathrm{B}$ and $\mathrm{pACTBD}-\mathrm{B}$. After pair-wise mating of the bait and prey strains and establishment of the diploid strains, interaction testing was undertaken by replica plating to selective media. Clear growth is seen for all vector combinations with the Bub1-Bub3 interaction, but differential growth is seen for the Mad1-Mad211 interaction. (B) Testing of the pGBAE-B and pACTBE-B vectors was undertaken by cloning four human genes in both plasmids and carrying out pair-wise matings. The protein interactions Cskn2b-Cskn2b, Cskn2b-Cskn2a2, and Atp6v1g1-Atp6v1e1 were reproduced as expected, although once again, variations in growth were noted. In this case, the apparent interaction strength was dependent upon whether Csnk2b was used as bait or prey. when secondary bands were visible cloning, an increase in the number of serts of incorrect size was found.

gap repair cloning experirelatively constant for each preparation of BamHI-digested vector. However, he number of white colonies was white coloctor. This suggests that of residual nonlinearized vectors following restriction enzyme digestion, which may vary between preparations. An exception occurred when attempts were made to clone as bait the mouse $\beta$-catenin gene, which encodes a protein with a transcriptional activation domain. Very few red colonies were observed, and all contained inserts of aberrant size. Similar analysis of the abundant white colonies demonstrated that most contained the correct insert, suggesting autoactivation of the $A D E 2$ reporter gene by the fusion protein, thereby making insertion undetectable by colony color. Such auto-activating baits are unsuitable for library screening and can be excluded at this early stage of bait construction.

It was also observed from a series of gap repair cloning experiments that although the number of background white colonies was usually consistent, the number of red colonies varied but correlated with the amount of insert DNA, as determined by band intensity of the PCR product. In general, the use of crude 
Table 1. Results of Parallel Yeast Two-Hybrid Screens Using Mouse Mad2/2 Protein as Bait

\begin{tabular}{|c|c|c|c|c|}
\hline Vector & $\begin{array}{l}\text { Accession No. } \\
\text { Matching IST }\end{array}$ & $\begin{array}{l}\text { Corresponding } \\
\text { Locus Name }\end{array}$ & Prey Description/Domains/Function & ISTs \\
\hline \multirow[t]{9}{*}{ pGBD-B } & NM_023429 & Ociad1 & Expressed in ES cells and developing vasculature & 18 \\
\hline & NM_025931 & Rabl4 & Putative ras-related GTP-binding protein, Rabl4 & 5 \\
\hline & XM_358819 & 9530039J15Rik & Noncoding $3^{\prime}$ UTR only & 2 \\
\hline & NM_178647 & Cggbp1 & Includes 300-bp 5' UTR & 2 \\
\hline & NM_011591 & Timm17b & Protein translocation across inner mitochondrial membrane & 1 \\
\hline & NM_026307 & 0610039D01Rik & Similar to proteins involved in divalent cation tolerance & 1 \\
\hline & NM_020493 & Srf & Noncoding 3' UTR only & 1 \\
\hline & XM_128800 & Map4k3 & Noncoding 3' UTR only & 1 \\
\hline & BC002262 & - & Single EST and genomic sequence match & 1 \\
\hline \multirow{6}{*}{ pGBAD-B } & NM_023056 & 1810009M01Rik & Lr8 protein & 2 \\
\hline & NM_145625 & Eif4b & Includes 700-bp 5' UTR & 1 \\
\hline & AK010603 & Atp6v1e1 & Noncoding 3' UTR only & 1 \\
\hline & $\mathrm{BC} 057660$ & Clta & Clathrin subunit involved in receptor-mediated endocytosis & 1 \\
\hline & BG296586 & Mm.328595 & Two EST and genomic sequence match & 1 \\
\hline & NM_011619 & Tnnt2 & Troponin T2, cardiac & 1 \\
\hline
\end{tabular}

PCR preparations directly in gap repair cloning produced more red colonies than when attempts were made to purify the DNA fragment, indicating that loss of DNA yield during purification more than outweighed any deleterious effect the components of the PCR may have on yeast transformation efficiency. Following this observation, gel purification of PCR products prior to cloning was undertaken only when significant secondary bands were observed. However, in such situations, the indicator vectors offer the most value in gap repair cloning, allowing easy identification of infrequent clones obtained from limiting amounts of DNA.

\section{Replication of Known Interactions}

The function of the insert indicator vectors pGBAD-B and pACTBD-B was initially tested. The coding regions of mouse Bub3 and Mad2ll genes were cloned as baits in pGBAD-B and pGBD-B, and appropriate fragments of mouse Bubl (codons 1-505) and Madl (codons 536-717) were cloned as preys in pACTBD-B and pACT2-B. This arrangement provides the ability to test for two known protein interactions from the spindle assembly checkpoint, Bub1-Bub3 (20) and Mad1-Mad211 (21), as well as providing negative interaction controls (Bub3-Mad1 and Mad211-Bub1). The expected interactions were confirmed, with no growth seen on the negative controls (Figure 3). The Mad1-Mad211 combinations resulted in less growth than the Bub1Bub3 combinations, suggesting a difference in interaction affinity, and the indicator vectors appeared slightly more sensitive at detecting the Mad1Mad211 interaction than non-indicator vectors. This effect was not so marked when the interactions were tested using the less stringent $H I S 3$ reporter gene.

The function of the pGBAE-B and pACTBE-B vectors was subsequently tested with two further well-characterized protein interactions-the first between the $\alpha^{\prime}$ and $\beta$ subunits of the human CK2 enzyme (encoded by $C S N K 2 A 2$ and $C S N K 2 B$, respectively) and the second between two subunits of the human vacuolar ATPase proton pump (encoded by ATP6V1G1 and ATP6V1E1). The CK2 enzyme is composed of four subunits: two regulatory $\beta$ subunits that homodimerize and two peripheral $\alpha$ or $\alpha^{\prime}$ catalytic subunits that interact with the $\beta$ subunit dimer (22). As expected, the Csnk2b protein interacted specifically with itself and with the Csnk2a2 protein in both orientations of bait and prey, although once again variations in growth were noted. In this case, the interaction strength was dependent upon whether Csnk2b was used as a bait or prey. In comparison, the protein subunits of the vacuolar ATPase proton pump, Atp6v1g1 and Atp6v1e1, demonstrated similar interaction in both orientations and did not interact with any of the other baits or preys tested (Figure 3).

\section{Function of Bait Vectors in Library Screens}

Although the new vectors were successful in replicating a small number of known interactions, the most demanding yeast two-hybrid application 
Table 2. Results of Parallel Yeast Two-Hybrid Screens Using Human Mccd1 Protein as Bait

\begin{tabular}{|c|c|c|c|c|}
\hline Vector & $\begin{array}{l}\text { Accession No. } \\
\text { Matching IST }\end{array}$ & $\begin{array}{l}\text { Corresponding } \\
\text { Locus Name }\end{array}$ & Prey Description/Domains/Function & $\begin{array}{l}\text { ISTs } \\
\text { (No.) }\end{array}$ \\
\hline \multirow{6}{*}{ pGBD-B } & NM_025072 & PTGES2 & Prostaglandin E synthase 2 & 9 \\
\hline & NM_003316 & TTC3 & Tetratricopeptide repeat domain 3, E3 ubiquitin ligase & 4 \\
\hline & NM_004270 & CRSP9 & Co-factor required for Sp1 transcriptional activation & 4 \\
\hline & NM_016223 & PACSIN3 & Protein kinase $\mathrm{C}$ and casein kinase substrate in neurons 3 & 2 \\
\hline & NM_001831 & CLU & Complement lysis inhibitor & 1 \\
\hline & NM_022909 & CENPH & Centromere protein $\mathrm{H}$ & 1 \\
\hline \multirow[t]{5}{*}{ pGBAD-B } & NM_025072 & PTGES2 & Prostaglandin E synthase 2 & 10 \\
\hline & NM_005051 & QARS & Glutaminyl-tRNA synthetase & 3 \\
\hline & NM_003316 & TTC3 & Tetratricopeptide repeat domain 3, E3 ubiquitin ligase & 1 \\
\hline & NM_004270 & CRSP9 & Co-factor required for Sp1 transcriptional activation & 1 \\
\hline & NM_022909 & CENPH & Centromere protein $\mathrm{H}$ & 1 \\
\hline \multirow[t]{6}{*}{ pGBAE-B } & NM_025072 & PTGES2 & Prostaglandin E synthase 2 & 7 \\
\hline & NM_003316 & TTC3 & Tetratricopeptide repeat domain 3, E3 ubiquitin ligase & 3 \\
\hline & NM_004663 & RAB11A & Member RAS oncogene family of small GTPases & 2 \\
\hline & NM_004270 & CRSP9 & Co-factor required for Sp1 transcriptional activation & 1 \\
\hline & NM_005051 & QARS & Glutaminyl-tRNA synthetase & 1 \\
\hline & NM_014608 & CYFIP1 & Cytoplasmic FMR1 interacting protein 1 & 1 \\
\hline
\end{tabular}

is library screening. It is possible that the presence of both the GAL4 activation and DNA binding domain sequences in the indicator vectors may predispose to false positives or that the decapeptide HPAFLYKVVD added to the $\mathrm{C}$ terminus of fusion proteins by pGBAE-B may increase the false negative rate. In an initial experiment, the full coding region of the mouse $M a d 2 l 2$ gene was cloned into the two bait vectors pGBAD-B and pGBD-B, and parallel screens were undertaken using a mouse day 7 embryo library (Matchmaker ${ }^{\mathrm{TM}}$; BD Biosciences Clontech). The analyses produced very similar results, with four genes identified in common between the two screens that together accounted for 50 out of a total of 64 clones analyzed (Table 1).
Finally, we carried out parallel screens with the recently described human Mccd1 protein (23) using bait vectors pGBD-B, pGBAD-B, and pGBAE-B across a human erythroleukemia library (Matchmaker). The bait was constructed by amplifying the $M C C D 1$ sequence from codon 25 to 119 , excluding the N-terminal hydrophobic mitochondrial localization sequence (23), because it causes promiscuous interaction in a yeast two-hybrid screen (24). Again, vectors performed consistently with each screen, identifying seven to eight interaction partners and a core of four preys in common between the three screens (Table 2). An additional five preys were identified in various combinations in at least two of the three screens, and one prey (Rab11a) was identified twice but in only one screen.

\section{DISCUSSION}

\section{Comparison of Indicator and Non-Indicator Vectors}

The indicator vectors clearly performed comparably to non-indictor vectors in the four previously reported interactions chosen for pair-wise testing, although some observations from this experiment are worth noting. The first of these is the apparent poor detection of the Mad1-Mad211 interaction for both classes of vector, suggesting a lower interaction affinity for this protein pair. This could reflect either a weaker physiological interaction for these two proteins, or it may be an artifact arising from the use of isolated protein fragments in a foreign environment. However, it did allow the direct comparison of vectors for their relative performance in detecting weak 
interactions, and both the pGBAD-B and $\mathrm{pACTBD}-\mathrm{B}$ indicator vectors were slightly more sensitive than their nonindicator counterparts. As the constructs are identical apart from the presence of a second GAL4 segment, it is not clear why this should be the case, although it is possible that the longer $3^{\prime}$ untranslated region of fusion transcripts from these vectors may result in increased protein expression.

A second observation of interest is the difference in reporter gene activation seen in the Csnk2a2-Csnk2b interaction, depending on which protein was expressed as a binding or activation domain fusion. Although this interaction was tested only with the pGBAE-B and pACTBE-B indicator vectors, we have observed more extreme examples of this phenomenon with other protein interactions and vectors (data not shown). Differential interference with interaction function produced by alternate domain fusions is likely to be protein-specific and may be one cause of false negative results in yeast two-hybrid screens.

\section{Vector Performance in Library Screens}

One of the potential problems with the new vectors in library screening is the possibility of reconstitution of GAL4 activity from bait constructs through spontaneous recombination, deletion, or base substitution. Such events may enhance the false positive rate among primary clones to an extent that the screens are overwhelmed, making the new vectors unusable. We routinely incorporate in our library screens a procedure of prey rescue and interaction reconfirmation, aimed at exclusion of a class of false positives that are not dependent upon interaction. Clones arising from reconstitution of GAL4 function in bait constructs should be excluded by this procedure, and significantly, baits constructed from the new vectors did not give rise to obviously higher numbers of excluded clones. This suggests that even if these events occur with the new bait vectors, the frequency is low enough to be accommodated in the standard screening procedure without a noticeable increase in effort.
Among the clones that remain after the reconfirmation procedure, it is not possible to formally exclude, without extensive further studies, false positives that could arise from nonphysiological interaction of fusion proteins in yeast. However, preys that were replicated between screens accounted for the majority of clones, demonstrating a similar overall performance of indicator and non-indicator vectors. The significance of preys that were restricted to just one of the vector screens is more difficult to evaluate with respect to vector function, but these were limited to clones identified only once or twice in any screen, suggesting that the depth of library screening is limiting rather than a systematic issue with false positive production or an increased false negative rate from the new vectors.

\section{New Interactors for the Mouse Mad212 and Human Mccd1 Proteins}

In addition to providing support for the performance of the new indicator vectors, the library screens undertaken have provided putative new interactions for the human Mccd1 protein and the mouse Mad212 protein that may assist functional characterization. Although further studies are required to validate these results, it seems that Mad212 is capable of interacting with a number of proteins and may be implicated in a wider range of cellular functions than previously suspected. The three screens undertaken with Mccd1 also produced generally consistent results that may assist with establishing a functional role for this protein.

Overall, the new indicator vectors performed comparably to standard vectors in common yeast two-hybrid applications. In addition, they can expedite the construction of baits and preys by significantly decreasing the effort involved in characterizing the products of gap repair cloning and should prove useful in high-throughput yeast two-hybrid projects requiring construction of large numbers of baits and preys.

\section{ACKNOWLEDGMENTS}

This work was supported by a University of Otago Research Grant, grants from the Otago Medical Research Foundation, the Health Research Council of New Zealand, and the UK Medical Research Council. We thank P. James for kindly providing the yeast host PJ69$4 A$ and plasmid $p G B D-C 1, I$. Herskowitz for the $p G A L-H O$ plasmid, and R.D. Gietz for the YCplac111 plasmid.

\section{COMPETING INTERESTS STATEMENT}

The authors declare no competing interests.

\section{REFERENCES}

1.Fields, S. and O. Song. 1989. A novel genetic system to detect protein-protein interactions. Nature 340:245-246.

2.Flajolet, M., G. Rotondo, L. Daviet, F. Bergametti, G. Inchauspe, P. Tiollais, C. Transy, and P. Legrain. 2000. A genomic approach of the hepatitis $\mathrm{C}$ virus generates a protein interaction map. Gene 242:369-379.

3.Rain, J.-C., L. Selig, H. De Reuse, V. Battaglia, C. Reverdy, S. Simon, G. Lenzen, F. Petel, et al. 2000. The protein-protein interaction map of Helicobacter pylori. Nature 409:211-215

4.Fromont-Racine, M., A.E. Mayes, A. Brunet-Simon, J.C. Rain, A. Colley, I. Dix, L. Decourty, N. Joly, et al. 2000. Genome-wide protein interaction screens reveal functional networks involving Sm-like proteins. Yeast 17:95-110.

5.Ito, T., T. Chiba, R. Ozawa, M. Yoshida, M. Hattori, and Y. Sakaki. 2001. A comprehensive two-hybrid analysis to explore the yeast protein interactome. Proc. Natl. Acad. Sci. USA 98:4569-4574.

6.Uetz, P., L. Giot, G. Cagney, T.A. Mansfield, R.S. Judson, J.R. Knight, D. Lockshon, V. Narayan, et al. 2000. A comprehensive analysis of protein-protein interactions in Saccharomyces cerevisiae. Nature 403:623-627.

7.Giot, L., J.S. Bader, C. Brouwer, A. Chaudhuri, B. Kuang, Y. Li, Y.L. Hao, C.E. Ooi, et al. 2003. A protein interaction map of Drosophila melanogaster. Science 302:17271736.

8.Li, S., C.M. Armstrong, N. Bertin, H. Ge, S. Milstein, M. Boxem, P.O. Vidalain, J.D. Han, et al. 2004. A map of the interactome network of the metazoan C. elegans. Science 303:540-543.

9.James, P., J. Halladay, and E.A. Craig. 1996. Genomic libraries and a host strain designed for highly efficient two-hybrid selection in yeast. Genetics 144:1425-1436.

10.Bendixen, C., S. Gangloff, and R. Rothstein. 1994. A yeast mating-selection scheme 
for detection of protein-protein interactions. Nucleic Acids Res. 22:1778-1779.

11.Bartel, P.L., J.A. Roecklein, D. SenGupta, and S. Fields. 1996. A protein linkage map of Escherichia coli bacteriophage T7. Nat. Genet. 12:72-77.

12.Finley, R.L., Jr. and R. Brent. 1994. Interaction mating reveals binary and ternary connections between Drosophila cell cycle regulators. Proc. Natl. Acad. Sci. USA 91:12980-12984.

13.Orr-Weaver, T.L., J.W. Szostak, and R.J. Rothstein. 1983. Genetic applications of yeast transformation with linear and gapped plasmids. Methods Enzymol. 101:228-245.

14.Ma, H., S. Kunes, P.J. Schatz, and D. Botstein. 1987. Plasmid construction by homologous recombination in yeast. Gene 58:201216.

15.Fusco, C., E. Guidotti, and A.S. Zervos. 1999. In vivo construction of cDNA libraries for use in the yeast two-hybrid system. Yeast 15:715-720.

16.Herskowitz, I. and R.E. Jensen. 1991. Putting the HO gene to work: practical uses for mating-type switching. Methods Enzymol. 194:132-146.

17.Ito, H., Y. Fukuda, K. Murata, and A. Kimura. 1983. Transformation of intact yeast cells treated with alkali cations. J. Bacteriol. 153:163-168.

18.Gietz, R.D. and A. Sugino. 1988. New yeastEscherichia coli shuttle vectors constructed with in vitro mutagenized yeast genes lacking six-base pair restriction sites. Gene 74:527534.

19.Petermann, R., B.M. Mossier, D.N. Aryee, and H. Kovar. 1998. A recombination based method to rapidly assess specificity of twohybrid clones in yeast. Nucleic Acids Res. 26:2252-2253.

20.Taylor, S.S., E. Ha, and F. McKeon. 1998 The human homologue of Bub3 is required for kinetochore localization of Bub1 and a Mad3/Bub1-related protein kinase. J. Cell Biol. 142:1-11.

21.Chen, R.H., A. Shevchenko, M. Mann, and A.W. Murray. 1998. Spindle checkpoint protein Xmad1 recruits Xmad2 to unattached kinetochores. J. Cell Biol. 143:283-295.

22.Gietz, R.D., K.C. Graham, and D.W. Litchfield. 1995. Interactions between the subunits of casein kinase II. J. Biol. Chem. 270:1301713021.

23.Semple, J.I., G. Ribas, G. Hillyard, S.E. Brown, C.M. Sanderson, and R.D. Campbell. 2003. A novel gene encoding a coiledcoil mitochondrial protein located at the telomeric end of the human MHC Class III region. Gene 314:41-54.

24.Lehner, B., J.I. Semple, S.E. Brown, D. Counsell, R.D. Campbell, and C.M. Sanderson. 2004. Analysis of a high-throughput yeast two-hybrid system and its use to predict the function of intracellular proteins encoded within the human MHC class III region. Genomics 83:153-167.
Received 2 December 2004; accepted 24 January 2005.

\section{Address for correspondence:}

David Markie

Pathology Department

Dunedin School of Medicine

University of Otago

P.O. Box 913

Dunedin, New Zealand

e-mail: david.markie@stonebow.otago.ac.nz 